\title{
A Subacute Rupture of the Posterior Inferior Cerebellar Artery Pseudoaneurysm after a Mild Traumatic Brain Injury
}

\author{
Xin Jin ${ }^{1 *}$, Jun Hua He ${ }^{1}$, Chen Hao Li ${ }^{1}$, Ding Kun Wang ${ }^{1}$ and Jin Jun Luo ${ }^{2^{*}}$ \\ ${ }^{1}$ Neurosurgery Department, Zhejiang Provincial Tongde Hospital, Hangzhou, Zhejiang, China \\ ${ }^{2}$ Departments of Neurology and Pharmacology, Temple University School of Medicine, Philadelphia, Pennsylvania, USA
}

Correspondence to:

Xin Jin, MD

Neurosurgery Department

Zhejiang Provincial Tongde Hospital

Hangzhou, Zhejiang, China

E-mail: jinxintd@126.com

Jin Jun Luo, MD, $\mathrm{PhD}$

Departments of Neurology and Pharmacology

Temple University School of Medicine

Philadelphia, Pennsylvania, USA

E-mail: jluo@temple.edu

Received: June 8, 2015

Accepted: August 2, 2015

Published: August 7, 2015

Citation: Jin X, He JH, Li CH, Wang DK, Luo JJ. 2015. A Subacute Rupture of the Posterior Inferior Cerebellar Artery Pseudoaneurysm after a Mild Traumatic Brain Injury. J Neurol Exp Neurosi 1(1): 6-9.

Copyright: (C) 2015 Jin et al. This is an Open Access article distributed under the terms of the Creative Commons Attribution 4.0 International License (CC-BY) (http://creativecommons. org/licenses/by/4.0/) which permits commercial use, including reproduction, adaptation, and distribution of the article provided the original author and source are credited.

Published by United Scientific Group

\begin{abstract}
The occurrence of intracranial traumatic pseudoaneurysm (TPA) in the posterior inferior cerebellar artery (PICA) is extremely rare. Management is challenging as it bears a high mortality. We encountered a case of PICA-TPA which was initially misdiagnosed as aneurysm pre-surgically. A 44-years old woman presented with sudden onset of change in mental status, headache and vomiting for 3 hours. She had a mild traumatic brain injury 6 weeks prior in a fall during which her left face hit the ground but no loss of consciousness or neurologic deficits. On examination, she exhibited stupor, GCS score $=9$, with nuchal rigidity and abnormal Kernig sign but no other focal findings. Brain CT showed hemorrhage in the $3^{\text {rd }}, 4^{\text {th }}$ and bilateral ventricles with a small amount of subarachnoid hemorrhage. CT angiography and conventional angiography with digital subtraction angiography showed an aneurysm of $1.5 \times 3.0 \times 4 \mathrm{~mm}$ in size with a width of neck in $3 \mathrm{~mm}$ attached to the right PICA. Craniotomy was performed during which an olive-shaped pseudoaneurysm, partially muscular, with blood clot, $10 \mathrm{~mm}$ in length and $6 \mathrm{~mm}$ in diameter adhered to the surrounding brain tissue was visualized. This pseudoaneurysm was suddenly separated free from PICA and caused vigorous bleeding during the procedure. Directly suturing the ruptured wall of the PICA successfully stopped bleeding and sealed the PICA. The patient recovered well, no neurological deficits were recognized at discharge to home one week post-surgery. Repeat cerebral angiography showed a normal PICA in shape and length.
\end{abstract}

\section{Keywords}

Posterior inferior cerebellar artery, Pseudoaneurysm, Rupture, Traumatic brain injury, Vascular reconstruction

\section{Introduction}

The incidence of intracranial traumatic pseudoaneurysm (TPA) is low which accounts for less than $1 \%$ of all intracranial aneurysms. Traumatic aneurysms are most often seen in artery segments of petrous or clinoid of internal carotid, cavernous sinus, middle or anterior cerebral arteries [1,2]. Most TPA are not a true aneurysm resulting from dissection causing separation between the intima, elastic lamina, middle and adventitia of the blood vessel. The pseudoaneurysmal wall usually consists of thrombus and tissue debris without a capsule or a neck. During surgery, a pseudoaneurysm is prone to be peeled off from the artery, ruptured and bled causing significantly debilitating morbidity and mortality. Moreover, surgical treatment for TPA is challenging with difficulty in approaching with high risk in morbidity and mortality $[1,3,4]$. There usually are three surgical approaches: 1 ) 
indirect approach which involves ligation or embolization of the diseased artery or anastomosis between intra- and extracranial arteries; 2) direct operation which clips or sutures the peusoaneurysm; and 3) an endovascular procedure using a stent or coil [5]. The occurrence of TPA in the posterior inferior cerebellar artery (PICA) is extremely rare. To our knowledge, there are only a few cases of PICA-TPA reported and no consensus on the treatment is seen in the literature [6]. We report here a case of PICA-TPA which was surgically managed successfully.

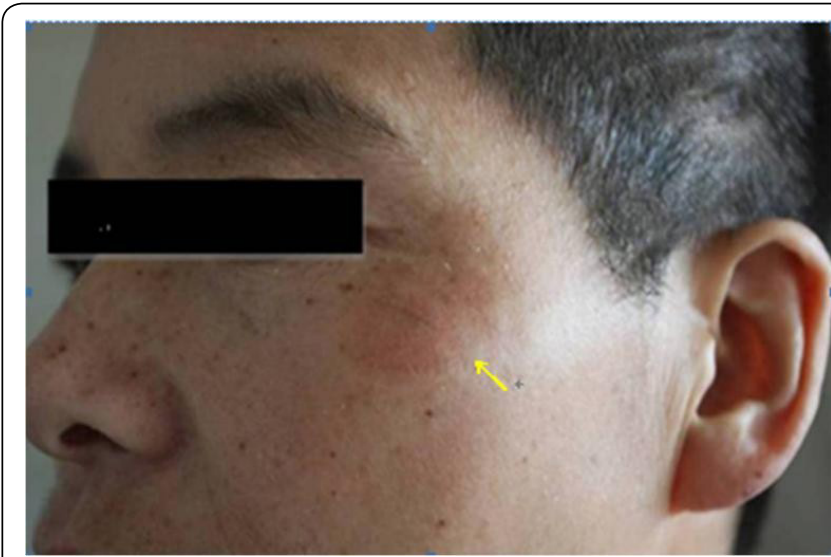

Figure 1: The arrow indicates a subsided bruise in the left zygomatic area from a fall 6 weeks ago.

\section{Case Report}

A 44-years old woman presented with sudden onset of headache, change in mental status and vomiting for 3 hours. Reportedly she had a mild traumatic brain injury (TBI) 6 weeks prior in a fall during which her left face hit the ground (figure 1) without loss of consciousness and without any neurologic deficits. On examination she exhibited stupor, GCS score $=9$, with nuchal rigidity, abnormal Kernig sign but no other focal findings. Brain CT showed hemorrhage
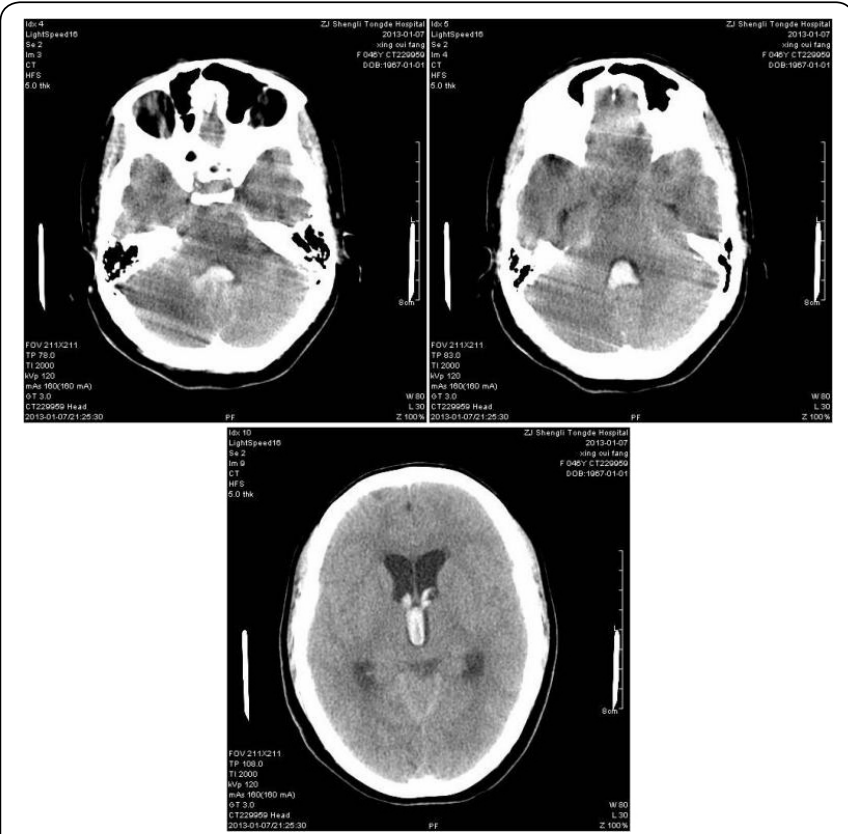

Figure 2: Pre-surgery brain CT showed hemorrhage into the 4th $[\mathrm{A}$ and $\mathrm{B}]$ and 3rd [C] ventricles [arrow]. in the $3^{\text {rd }}, 4^{\text {th }}$ and bilateral ventricles with a small amount of subarachnoid hemorrhage [Hunt-Hess scale = 4] (figure 2). An initially provisional diagnosis of spontaneous intraventricular hemorrhage was made. She was admitted into the intensive care unit.

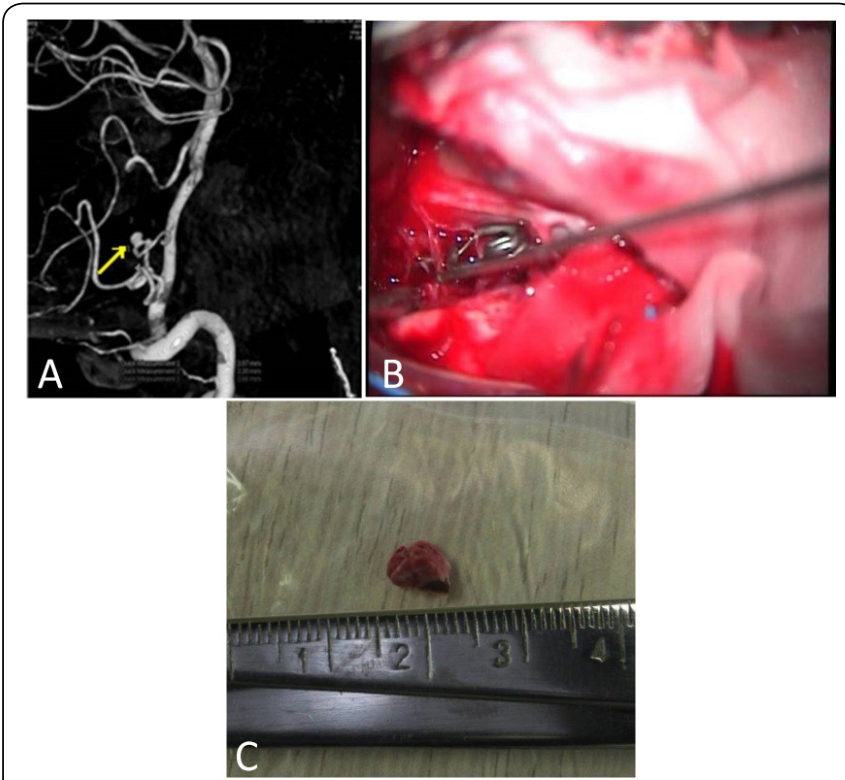

Figure 3: Pre-surgery conventional angiography with DSA showed a PICA aneurysm [arrow, A].Transiently clipping PICA and suturing the ruptured vessel wall was shown $[\mathrm{B}]$. Pseudoaneurysm after formalin-fixation [shrunk in volume, $\mathrm{C}]$.

Her mental status improved 12 hours after admission. Repeat brain CT scan showed a reduced amount of intraventricular hemorrhage while CT angiography (CTA) showed a suspicious cerebellar artery aneurysm which was confirmed by conventional with digital subtraction angiography (DSA) showing an aneurysm of $1.5 \times 3.0 \times 4 \mathrm{~mm}$ in size with a neck of $3 \mathrm{~mm}$ in width attached to the right PICA (figure $3 \mathrm{~A}$ ). Considering the size, shape, and location of the aneurysm, and the beneficial and adverse effects of treatment, the option of open surgical procedure to clip was chosen.

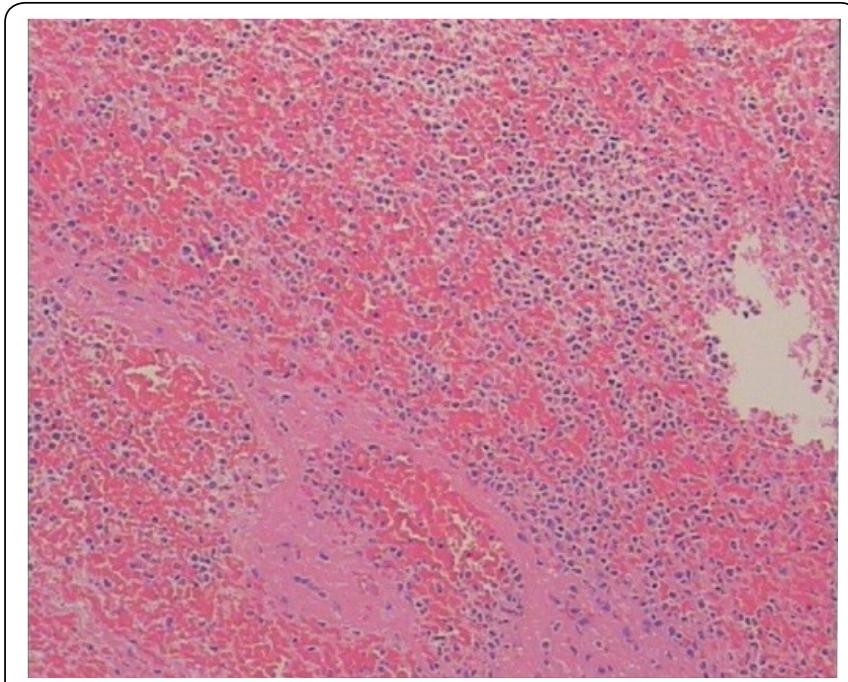

Figure 4: Histological study (H.E. staining) showed neutrophils and lymphoplasmacytic infiltration with necrosis consistent with a pseudoaneurysm. 


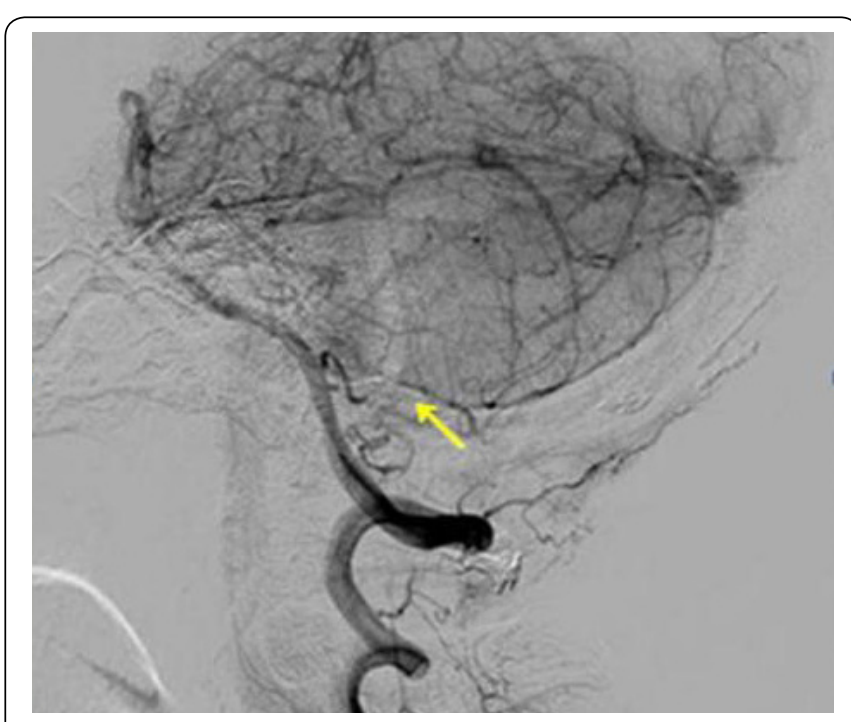

Figure 5: Post-surgery conventional angiography with DSA showing a patent right PICA. The previously recognized "aneurysm" is no longer seen.

After opening the foramen magnum and posterior arch ring via the posterior midline approach, it was noted that subarachnoid hemorrhage in the posterior fossa was insignificantly visible. Under adequate exposure, the right PICA was visualized as $2 \mathrm{~mm}$ in diameter originating from the right vertebral artery and tortuously traveling towards the ventrolateral aspect of the brainstem. An olive-shaped, partially muscular, blood clot, $10 \mathrm{~mm}$ in length and $6 \mathrm{~mm}$ in diameter adhering to the surrounding brain tissue and arachnoid emerged, as the one that was initially misimpressed as the PICA aneurysm by CTA and conventional angiogram. During the exploring procedure on the right PICA along the whole bump of the "aneurysm", the "mass" suddenly separated free and a V-shaped crack appeared on the PICA (figure 3C). As simply clipping PICA to stop bleeding would cause ischemia and possible infarction in the PICA territory, direct suturing the ruptured PICA was performed using 9-0 Prolene suture which successfully sealed the PICA (figure 3B). Histological study on the separated mass showed neutrophils and lymphoplasmacytic infiltration with necrosis consistent with a pseudoaneurysm (figure 4). Postsurgical conventional cerebral angiography with DSA showed a normal PICA in shape and length. The previously recognized "aneurysm" was no longer seen (Figure 5). A week post-operation, the patient who recovered well with no neurological deficits was discharged home.

\section{Discussion}

To our knowledge the incidence of PICA-TPA is extremely rare. A PubMed searching showed only 9 cases in total which were pooled from 7 articles for the period from 1985 to 2015 [6-12]. Among those cases, all had intraventricular or subarachnoid hemorrhage, 6 with TBI and 4 of them had a skull fracture, 5 with transient loss of consciousness, and only 1 without obvious TBI, fracture or loss of consciousness. Apparently, there was a relationship between the TBI and the occurrence of PICA-TPA. Fractures in the slope and occipital areas were recognized as the most frequent injury in locations with the occurrence of PICA-TPA in the posterior circulation which had been confirmed by craniotomy in 4 out of 5 [6-12]. The intracranial vascular vessels lack the external elastic layer and have a thin inner smooth muscular layer. Approximately $15 \%-25 \%$ of the aneurysms are caused by an open head injury while $70 \%$ by a closed head injury directly or indirectly impact blood vessels. Accelerating or decelerating brain injury may shear and tear dura and blood vessel as the brain and the skull moves at different speeds relative to the motion generators [1-4], particularly in the segment of the internal carotid artery in petrous or clinoid locations, or vertebral, basilar, PICA arteries, or cavernous sinus. Occurrence of TPA usually results from intracranial vascular injury triggered by external forces.

Several clinical manifestations may suggest a plausible diagnosis of TPA after TBI, such as 1) delayed neurological deterioration; 2) concomitant subarachnoid hemorrhage; 3) a large intracerebral hematoma; and 4) unexplainable bleeding during the evacuation of an intracranial hematoma. Clinical features of the PICA-TPA may include a delayed onset of headache, change in mental status, and intracranial hemorrhage into the fourth, and sometime the third and lateral, ventricle. Rupture of a TPA may occur mostly 2-3 weeks after a TBI, however, it may occur as early as a few hours or delayed after 10 years $[1,8,13]$. Brain CT and angiography imaging are valuable tools to initially identify if there is a TPA in patients with intracerebral hemorrhage. Occasionally a negative brain CT angiography can be encountered due to vasospasm and the small caliber PICA. Brain MRI may provide additional value in evaluation of the pseudoaneurysm, particularly for those perivascular in location with a round or irregular shape and larger in diameter than the abnormal signals for an aneurysm displayed on cerebral angiography $[1,2]$. Diagnosis can be made with a conventional cerebral angiography with 3D-DSA which is considered the gold standard in making diagnosis. It may show an irregular contour with round, gourd-like, or teardrop in shape of the TPA. A delay in filling and emptying of the contrast agent is often evident. Rarely, missed diagnosis may occur such as seen in our case.

Our case was unique as the patient only suffered a mild TBI without loss of consciousness or presence of focal neurologic deficits for up to 6 weeks. Findings of the presenting symptoms with sudden onset of headache, change in mental status and vomiting for 3 hours and neurologic examination showing nuchal rigidity and abnormal Kernig sign, all indicated the signs of presence of an increased intracranial pressure in this patient, possibly due to small bleeding, leaking into the posterior fossa from the pseudoaneurysm. A visible bruise on her left zygomatic area from the fall was realized on admission (Figure 1). Her clinical manifestations were consistent with those of PICA-TPA. However, the correct diagnosis was not made until during the craniotomy as the initial cerebral angiography misinterpreting a PICA aneurysm. No MRI was performed in our case. No fracture or tearing dura or vessels, or structural lesions in the brainstem and cerebellum were found in craniotomy. Unexpected separation of the PICATPA produced a V-type crack in the PICA causing massive bleeding which was surgically repaired successfully.

The mortality of a ruptured pseudoaneurysm is high as reported 32\%-50\%, while surgical intervention decreased it to 
less than $10 \%[1,4]$. The mortality of PICA-TPA is unknown but may be higher due to its anatomic location. Differential diagnosis including pseudoaneurysm should be exercised in TBI patients. Surgical options for treating traumatic aneurysm include indirect or direct approaches and endovascular procedures $[2,4]$. The mortality of those performances are high because majority of traumatic aneurysm are pseudoaneurysm in nature, and the aneurysmal wall is fragile and consists of injured smooth muscle layer of the blood vessel and neural tissues, which are prone to rupture $[1,2,4]$. Therefore, surgical approaches are preferred [4]. Direct surgery with clipping yields a low efficacy and difficulty in reconstruction of the artery plasticity and a high rate of the re-stenosis after the procedure [14]. Endovascular therapy has been increasingly used recently in treatment of aneurysms but remains a challenge in managing TPA as it is prone to rupture if using coil embolization. Notably, some aneurysms may rupture after embolization. While a stent and coil embolization in combination is a better way for treating a larger vessel aneurysm $[5,14,15]$. Treatment for PICA-TPA should be individualized based on the size and location on PICA. There are five areas that had been suggested for comparison in surgical outcomes: front medulla, lateral medulla, posterior medulla, cerebellar tonsils, cerebellar hemispheres and vermis. Unfortunately, no satisfactory benefits have been reported [16]. O'Schuster and colleagues reported three cases of PICA-aneurysms in medulla oblongata receiving clipping procedures. One of the three with an aneurysmal neck recovered well while the other 2 without a neck had brainstem symptoms [16]. O'Shaughnessy and colleagues reported a case of the PICA pseudoaneurysm at the initial bifurcation location, an anastomosis between the PICA and vertebral artery was performed and achieved a clinical better outcome [17].

In our PICA-TPA case, rupture of the PICA involving the lateral medulla oblongata could not be ligated. Microclipping was employed temporarily to stop bleeding while suturing on the ruptured PICA was carried out successfully to sustain PICA irrigation. Similarly, a technique to repair a tear at the base of a blister-like aneurysm was once previously recommended using suturing and an encircling clip [18]. Actually, suturing the ruptured atrial vessel is a relatively simple procedure and easy to perform. It may be a choice of options in treating PICA-TPA.

\section{Conclusion}

PICA-TPA is extremely rare. Clinical manifestations of PICA-TPA may include a delayed onset of deteriorating neurologic symptoms with headache, change in mental status, and the signs of increased intracranial pressure. PICATPA may result from TBI with posterior fossa fracture, or even a mild TBI without initial clinical symptoms. Cerebral angiography confirms the diagnosis but a missed diagnosis may occur. Treatment options may include direct and indirect surgery and an endovascular procedure. Suturing the ruptured vessel may be beneficial.

\section{References}

1. Larson PS, Reisner A, Morassutti DJ, et al. 2000. Traumatic intracranial aneurysms. Neurosurg Focus 8(1).

2. Stallmeyer MJ, Morales RE, Flanders AE. 2006. Imaging of traumatic neurovascular injury. Radiol Clin North Am 44(1):13-39. doi: 10.1016/j. rcl.2005.08.003

3. Krings T, Geibprasert S, terBrugge KG. 2010. Pathomechanisms and treatment of pediatric aneurysms. Childs Nerv Syst 26(10): 1309-1318. doi: 10.1007/s00381-009-1054-9

4. Jafar JJ, Huang PP. 1998. Surgical treatment of carotid cavernous aneurysms. Neurosurg Clin NAm 9(4): 755-763.

5. Hemphill JC, Gress DR, Halbach W. 1999. Endovascular therapy of traumatic injuries of the intracranial cerebral arteries. Critical care clinics 15(4): 811-829. doi: 10.1016/S0749-0704(05)70089-0

6. Morard M, de Tribolet N. 1991. Traumatic aneurysm of the posterior inferior cerebellar artery: case report. Neurosurgery 29(3): 438-441.

7. Hossain M, Brown J, McLean AN, et al. 2002. Delayed presentation of post-traumatic aneurysm of the posterior inferior cerebellar artery in a patient with spinal cord injury. Spinal Cord 40(6): 307-309. doi: 10.1038/sj.sc.3101290

8. McLaughlin N, Bojanowski MW. 2005. Ruptured aneurysm at the choroidal branch of the posterior inferior cerebellar artery: a case report, review of the literature and proposed pathogenesis. BrJNeurosurg 19(3): 250-253. doi: $10.1080 / 02688690500208460$

9. Sure U, Becker R, Petermeyer M, et al. 1999. Aneurysm of the posterior inferior cerebellar artery caused by a traumatic perforating artery tearout mechanism in a child. Childs Nerv Syst 15(6-7): 354-356. doi: $10.1007 / \mathrm{s} 003810050411$

10. Purgina B, Milroy CM. 2015. Fatal traumatic aneurysm of the posterior inferior cerebellar artery with delayed rupture. Forensic Sci Int 247: e1-5. doi: 10.1016/j.forsciint.2014.11.003

11. Binning MJ, Hauschild TB, Amini A, et al. 2009. Delayed posttraumatic saccular aneurysm of PICA in an adolescent. Acta Neurochir (Wien) 151(12):1647-1648. doi: 10.1007/s00701-009-0323-4

12. Meguro K, Rowed DW. 1985. Traumatic aneurysm of the posterior inferior cerebellar artery caused by fracture of the clivus. Neurosurgery 16(5): 666-688.

13. Proust F, Callonec F, Bellow F, et al. 1997. Tentorial edge traumatic aneurysm of the superior cerebellar artery. Case report. J Neurosurg 87(6): 950-954.

14. Maras D, Lioupis C, Magoufis G, et al. 2006. Covered stent-graft treatment of traumatic internal carotid artery pseudoaneurysms: a view. Cardiovasc Intervent Radiol 29(6): 958-968. doi: 10.1007/s00270-0050367-7

15. Fujimura M, Nishijima M, Midorikawa H, et al. 2003. Fatal rupture following intra-aneurysmal embolization for the distal posterior inferior cerebellar artery aneurysm with parent artery preservation. Clin Neurol Neurosurg 105(2): 117-120. doi: 10.1016/S0303-8467(02)00131-2

16. Schuster JM, Santiago P, Elliott JP, et al. 1999. Acute traumatic posteroinferior cerebellar artery aneurysms: report of three cases. Neurosurgery 45(6): 1465-1467.

17. O'Shaughnessy BA, Getch CC, Bowman RM, et al. 2005. Ruptured traumatic vertebral artery pseudoaneurysm in a child treated with trapping and posterior inferior cerebellar artery reimplantation. Case report and review of the literature. J Neurosurg 102(2 Suppl): 231-237.

18. Yanaka K, Meguro K, Nose T. 2002. Repair of a tear at the base of a blister-like aneurysm with suturing and an encircling clip: technical note. Neurosurgery 50(1): 218-221. 